\title{
SOLIDIFICAÇÃO UNIDIRECIONAL TRANSITÓRIA, MICROESTRUTURA, PARÂMETROS TÉRMICOS E DUREZA DE LIGAS AA5052 VARIANDO TEOR DE Mg*
}

Cínthia Toshimi Ogata José Eduardo Spinelli

\section{Resumo}

Processos de fundição de ligas de alumínio com altos teores de Mg são um desafio da indústria, uma vez que diferentes taxas de resfriamento ocorrem em geometrias diversas. Este trabalho tem como objetivo caracterizar a liga de alumínio AA5052 com variações em teores de $\mathrm{Mg}$ por meio de experimentos de solidificação direcional transitória, e compreender seus efeitos com relação a evolução de parâmetros térmicos, microestruturais, e propriedade mecânica. As leis experimentais de evolução do espaçamento dendrítico primário $\left(\lambda_{1}\right)$ com relação a taxa de resfriamento resultam em: $\lambda_{1}=640{\dot{T}_{L}}^{-1 / 4}$ para a liga 5052 com $2,4 \% \mathrm{Mg} \mathrm{e}$ $\lambda_{1}=300 \dot{T}_{L}^{-1 / 4}$ para a liga com $3,2 \% \mathrm{Mg}$. As leis de crescimento de espaçamento dendrítico secundário $\left(\lambda_{2}\right)$ com relação a velocidade de solidificação resultaram em: $\lambda_{2}=27 V_{L}^{-1,1}$ para a liga $5052 \mathrm{com} 2,4 \% \mathrm{Mg}$ e $\lambda_{2}=15 V_{L}^{-1,1}$ para as ligas com $2,6 \% \mathrm{Mg}$ e $3,2 \% \mathrm{Mg}$. Maiores teores de $\mathrm{Mg}$ na liga AA5052 proporcionaram uma expressiva redução no valor de $\lambda_{2}$ para uma determinada $V_{\mathrm{L}}$. A evolução da dureza mostrou-se diretamente relacionada ao tamanho e a distribuição dos intermetálicos presentes, sendo $\lambda_{2}$ o fator predominante neste controle.

Palavras-chave: Liga AA5052; Solidificação Direcional; Thermo-Calc; Intermetálicos; Microestrutura.

\section{TRANSIENT DIRECTIONAL SOLIDIFICATION, MICROSTRUCTURE, SOLIDIFICATION THERMAL PARAMETERS AND HARDNESS OF AA5052 ALLOY VARYING MG CONTENT}

\section{Abstract}

Casting process of high-Mg content Aluminum Alloys is considered an industry challenge since different cooling rates occur during solidification of complex geometries. The present research aims to characterize the commercial aluminum AA5052 alloy with different $\mathrm{Mg}$ contents through transient directional solidification experiment. The results allowed to comprehend the effect of $\mathrm{Mg}$ content with respect to thermal parameters, microstructural parameters and mechanical properties (Brinell hardness). The experiments reached cooling rates between 7.0 and $30.0^{\circ} \mathrm{C} / \mathrm{s}$ (considering the closest thermocouple from the bottom of the casting, i.e., $3 \mathrm{~mm}$ ). The experimental growth law for microstructural evolution of $\lambda_{1}$ for the different chemical compositions were obtained, resulting in: $\lambda_{1}=640 \dot{T}_{L}^{-1 / 4}$ to the 5052 alloy with $2.4 \mathrm{wt} . \% \mathrm{Mg}$ and $\lambda_{1}=300 \dot{T}_{L}^{-1 / 4}$ to the examined samples with $3.2 \mathrm{wt} . \% \mathrm{Mg}$. Secondary dendritic growth laws were also obtained concerning their variation with the growth rates, resulting in the following expressions: $\lambda_{2}=27 V_{L}^{-1.1}$ for the 5052-2.4wt.\%Mg and $\lambda_{2}=15.0 V_{L}^{-1.1}$ for the 5052-2.6wt.\%Mg and 5052-3.2wt.\%Mg alloys. Thus, 5052 alloys containing higher Mg content may allow a significant decrease on $\lambda_{2}$ value for a certain $V_{L}$. The hardness values were directly related to the size and distribution of the intermetallic particles and showed $\lambda_{2}$ as the main variable to affect mechanical strength of the tested alloys.

Keywords: AA5052 alloy; Directional Solidification; Thermo-Calc; Intermetallic; Microstructure.

Mestranda em Eng. de Materiais DEMa/UFSCar, Graduação em Eng. De Materiais DEMa/UFSCar (2014).

Professor Adjunto do DEMa/UFSCar. Doutor em Engenharia Mecânica (UNICAMP-FEM-DEMA/2005). Mestre em Engenharia Mecânica (UNICAMP-FEM-DEMA/2000). 


\section{INTRODUÇÃO}

O teor de Mg em ligas de alumínio da série 5xxx varia entre 0,5-5\% em massa e impacta diretamente na formação de intermetálicos e principalmente nas propriedades mecânicas do produto final, uma vez que este é o principal elemento endurecedor por solução sólida. Em termos de processamento de tais ligas em seu estado fundido, a dificuldade de vazamento aumenta quanto maior for o teor de magnésio. $\mathrm{O} \mathrm{Mg}$ facilmente oxida-se ao longo da operação do banho líquido o que dificulta o controle estequiométrico da composição química. Além disso aumenta o intervalo de solidificação da liga, o que dificulta o controle da mushy zone quando a solidificação ocorre de maneira contínua e em geometrias complexas ou limitadas. Explicitada a importância e desafio a respeito do vazamento de ligas de alumínio da série $5 x x x$, soma-se a escassez de trabalhos na literatura envolvendo análise de correlação entre microestrutura e parâmetros térmicos de ligas à base de $\mathrm{Al}-\mathrm{Mg}$. $\mathrm{O}$ presente trabalho tem como objetivo principal compreender os efeitos da variação de Mg na evolução de parâmetros térmicos (taxa de resfriamento e velocidade de solidificação), parâmetros microestruturais (espaçamento dendrítico primário - $\lambda_{1} \mathrm{e}$ secundário $-\lambda_{2}$ ), e variação de dureza Brinell, através de ensaio de solidificação unidirecional da liga 5052 com teores 2,4, 2,6 e 3,2\%Mg, identificados por 5052$2,4 \% \mathrm{Mg}, 5052-2,6 \% \mathrm{Mg}$ e 5052-3,2\%Mg. São incluídos também como objetivos secundários a comparação qualitativa entre as microestruturas encontradas (formação de intermetálicos) e verificação da funcionalidade de leis experimentais de crescimento do tipo $\lambda_{1}=\mathrm{a}(\dot{T})^{-0,55}$ e $\lambda_{2}=\mathrm{b}(\mathrm{V})^{-2 / 3}$ (tipicamente empregadas para ligas binárias de $\mathrm{Al}$ ) para as ligas comerciais estudadas.

A importância do estudo do processo de solidificação e os parâmetros que influenciam em sua dinâmica (temperatura de vazamento, geometria do molde, tipo de molde) são extremamente importantes uma vez que a estrutura formada após a solidificação determina as propriedades do produto final.

Os principais dispositivos utilizados para estudo e compreensão de solidificação unidirecional em fluxo estacionário de calor (independente do tempo) são os fornos Bridgman, na qual o metal líquido dentro de um cadinho é movimentado dentro de um forno com regiões em temperaturas diferentes, isto é, movimentado sob um gradiente de temperatura, sendo assim possível controlar o crescimento dos grãos e do arranjo dendritico [1]. Para reprodução de situações reais, dispositivos de extração de calor em regime transitório são mais utilizados, sendo capazes de oferecer taxas de resfriamento entre 0,1 e $150^{\circ} \mathrm{C} / \mathrm{s}$ dependendo da liga empregada $\mathrm{e}$ condições do molde (material e acabamento superficial) [2] [3].

Alguns modelos teóricos para crescimento dendrítico foram reportados nas últimas décadas, sendo os mais conhecidos e de maior aplicabilidade os Modelos de Hunt-Lu [4] e de Bouchard e Kirkaldy [5]. Esses estudos foram capazes de estabelecer relações entre parâmetros estruturais e parâmetros térmicos de solidificação apresentadas na forma generalizada pela equação abaixo:

(Parâmetro microestrutural $)=c(\text { parâmetro térmico })^{-b}$

Equação 1

Os parâmetros microestruturais podem ser espaçamentos celulares $\left(\lambda_{c}\right)$, dendríticos primários $\left(\lambda_{1}\right)$, secundários $\left(\lambda_{2}\right)$, terciários $\left(\lambda_{3}\right)$, e eutéticos ou interfásicos $(\lambda)$. Já os parâmetros térmicos são gradiente de temperatura $(G)$, velocidade de solidificação (V) e taxa de resfriamento (Tं). 'c' é uma constante que depende da composição química da liga, e 'b' é um expoente determinado experimentalmente. 
Bouchard e Kirkaldy [5] desenvolveram um modelo que denominaram de semiempírico, correlacionando o espaçamento dendrítico primário com os parâmetros térmicos de solidificação para condições de extração de calor em regime transitório, incorporando a racionalidade e os parâmetros constitutivos dos modelos teóricos desenvolvidos para condições estacionárias de fluxo de calor [6-8]. Este modelo foi comprovado por Quaresma e co-autores por meio de um estudo envolvendo a soldificação de ligas Al-Cu [9].

Para condições de fluxo de calor transitório, foram determinadas relações experimentais para o crescimento dendritico primário de uma série de sistemas metálicos (ligas binárias) do tipo:

$\lambda_{1}=K\left(G_{L} V\right)^{-a}$

Equação 2

$\mathrm{Na}$ Tabela 1 abaixo, segue uma série de fatores de potência da lei de crescimento dendrítico primário para ligas binárias de alumínio, em solidificação em condições de fluxo de calor transitório.

Tabela 1- Fatores exponenciais das leis de crescimento dendrítico primário para ligas metálicas na solidificação em condições de fluxo de calor transitório [10][11].

\begin{tabular}{|c|c|}
\hline Liga & a \\
\hline $\mathrm{Al}-4,0 \% \mathrm{Cu}$ & 0,45 \\
\hline $\mathrm{Al}-10,0 \% \mathrm{Cu}$ & 0,57 \\
\hline $\mathrm{Al}-1,1 \% \mathrm{Si}$ & 0,56 \\
\hline $\mathrm{Al}-2,8 \% \mathrm{Si}$ & 0,57 \\
\hline $\mathrm{Al}-0,5 \% \mathrm{Ni}$ & 0,48 \\
\hline $\mathrm{Al}-3 \% \mathrm{Mg}$ & 0,55 \\
\hline $\mathrm{Al}-6,5 \% \mathrm{Mg}$ & 0,55 \\
\hline $\mathrm{Al}-6,5 \% \mathrm{Mg}-1 \% \mathrm{Si}$ & 0,55 \\
\hline
\end{tabular}

No caso dos espaçamentos secundários, tanto para solidificação em condições de fluxo de calor estacionário quanto para transitório, a literatura mostra um único valor médio para o fator exponencial $a$, qual seja, $a=0,34$.

Bouchard e Kirkaldy [5] também desenvolveram uma expressão para o cálculo do espaçamento dendrítico secundário, porém desconsiderando o fenômeno de engrossamento dos braços secundários, apresentando com única variável a velocidade de deslocamento da ponta da dendrita que é dada por:

$\lambda_{2}=2 a_{2} \pi\left[\frac{4 \sigma_{S L}}{C_{0}(1-k)^{2} L_{V}}\left(\frac{D_{L}}{v_{L}}\right)^{2}\right]^{1 / 3}$

Equação 3

Onde: $\sigma s \mathrm{~s}$ é a tensão superficial sólido-líquido, Dı é o coeficiente de difusão de soluto no líquido, $\mathrm{C}_{0}$ é a composição nominal, $\mathrm{k}$ é uma constante adimensional, $\mathrm{L}_{v}$ é o calor latente na base volumétrica $\left(\mathrm{J} / \mathrm{m}^{3}\right)$ e a2, um fator de calibração usado para ajustar os desvios nos valores de difusividade térmica e de $\lambda_{2}$ [10].

O levantamento de equações que relacionem parâmetros microestruturais com propriedades mecânicas (limite de escoamento, limite de resistência a tração, ductilidade, limite de resistência a fadiga e fluência) é extremamente útil em termos práticos para a engenharia uma vez que auxiliam na busca de uma forma de programação estrutural por meio da fixação prévia das condições de solidificação em função de um determinado nível de resistência mecânica que se pretenda alcançar. 
A equação de Hall-Petch, por exemplo, correlaciona o tamanho médio dos grãos com o limite de escoamento do material, segundo a expressão abaixo:

$\sigma_{e}=\sigma_{0}+K d^{-1 / 2}$

Equação 4

Onde $\mathrm{K}$ é constante que representa a medida da extensão do empilhamento de discordâncias, $\sigma_{0}$ é a tensão de atrito que se opõe ao movimento das discordâncias e d é o diâmetro do grão.

Reconhece-se atualmente que os espaçamentos dendríticos possuem uma correlação muito mais estreita com as propriedades mecânicas do que o próprio tamanho de grão. No interior de cada grão existe uma rede de ramificações dendríticas caracterizada por ter baixas concentrações de soluto no centro das ramificações e altas concentrações nos interstícios (considerado coeficiente de redistribuição $<1$, que é o caso do Al-Mg), podendo haver até mesmo precipitação de fases e compostos intermetálicos. Além disso, existindo a presença de gases dissolvidos no metal líquido, as regiões interdendríticas são particularmente mais adequadas a fixação de bolhas. Adicionalmente aos obstáculos intergranulares ao escorregamento, existirão obstáculos distribuídos pela rede dendrítica internamente ao grão cristalino. Tanto para aplicações com a estrutura bruta de fusão como para estruturas que serão submetidas a tratamentos térmicos posteriores (solubilização e precipitação) é importante o grau de refino da estrutura dendrítica. Para estruturas brutas de fusão, a distribuição uniforme de outras fases e outros obstáculos de escorregamento favorece as propriedades mecânicas. Para estruturas que sofrerão tratamento posterior, esta condição favorece a redução de tempo de tratamento.

Dessa forma, seria extremamente interessante dispor de expressões que correlacionassem parâmetros de estrutura dendrítica $\left(\lambda_{1}\right.$ e $\left.\lambda_{2}\right)$ com propriedades mecânicas na forma:

$H B, \sigma$ ou $\delta=f\left(\lambda_{1}, \lambda_{2}\right)$

Equação 5

Onde $\sigma$ é uma tensão característica do campo plástico do material, $\delta$ uma característica de sua ductilidade, como alongamento e HB, valor de dureza Brinell.

\section{MATERIAIS E MÉTODOS}

Para a realização deste estudo será adotada uma metodologia com as seguintes etapas de desenvolvimento:

a) Solidificação unidirecional vertical ascendente das 3 ligas ASTM 5052 com variações em composição química, em regime transiente de extração de calor, cujo dispositivo está ilustrado na Figura 1;

b) Verificação da composição química ajustada em cada uma das amostras propostas e equipamento de Espectroscopia de Emissão Ótica (ARL-Applied Research Laboratories, modelo 3640);

c) Determinação de parâmetros térmicos da solidificação transitória a partir das curvas registradas pelos termopares tipo $\mathrm{K}$ posicionados em diferentes posições do lingote ( $V_{L}$ - velocidade da isoterma liquidus ,e $\dot{T}^{-}$- taxa de resfriamento);

d) Corte do lingote solidificado unidirecionalmente para caracterização macro e microestrutural ao longo de seu comprimento (espaçamentos dendríticos primários e secundários). Para tanto, será empregada a técnica de microscopia ótica com luz polarizada. A medição dos espaçamentos dendríticos foram realizadas com auxílio do software ImageJ e quantificadas através do método dos triângulos [12].

e) Determinação de leis experimentais de crescimento através das correlações entre os parâmetros térmicos e microestruturais obtidos; 
f) Avaliação dos efeitos da variação do teor de Mg tanto nos parâmetros térmicos quanto na microestrutura formada.

g) Realização de cálculos termodinâmicos com software Thermo-Calc no intuito de estudar a formação de intermetalicos em condições fora de equilíbrio. A análise de algumas amostras em MEV (microscópio eletrônico de varredura (FEI Inspect S 50), e EDS (Espectroscopia de Energia Dispersiva) será realizada para complementar a caracterização dos intermetálicos.

h) Realização de ensaios de dureza Brinell (com base na norma ASTM E10 - 15a Standard Tetst Method for Brinell Hardness of Metallic Materials) em diferentes posições do lingotes correspondentes as 3 ligas estudadas, quais sejam, as ligas $5052-2,4 \% \mathrm{Mg}$, $5052-2,6 \% \mathrm{Mg}$ e $5052-3,2 \% \mathrm{Mg}$. Levantamento dos perfis experimentais em função da microestrutura.
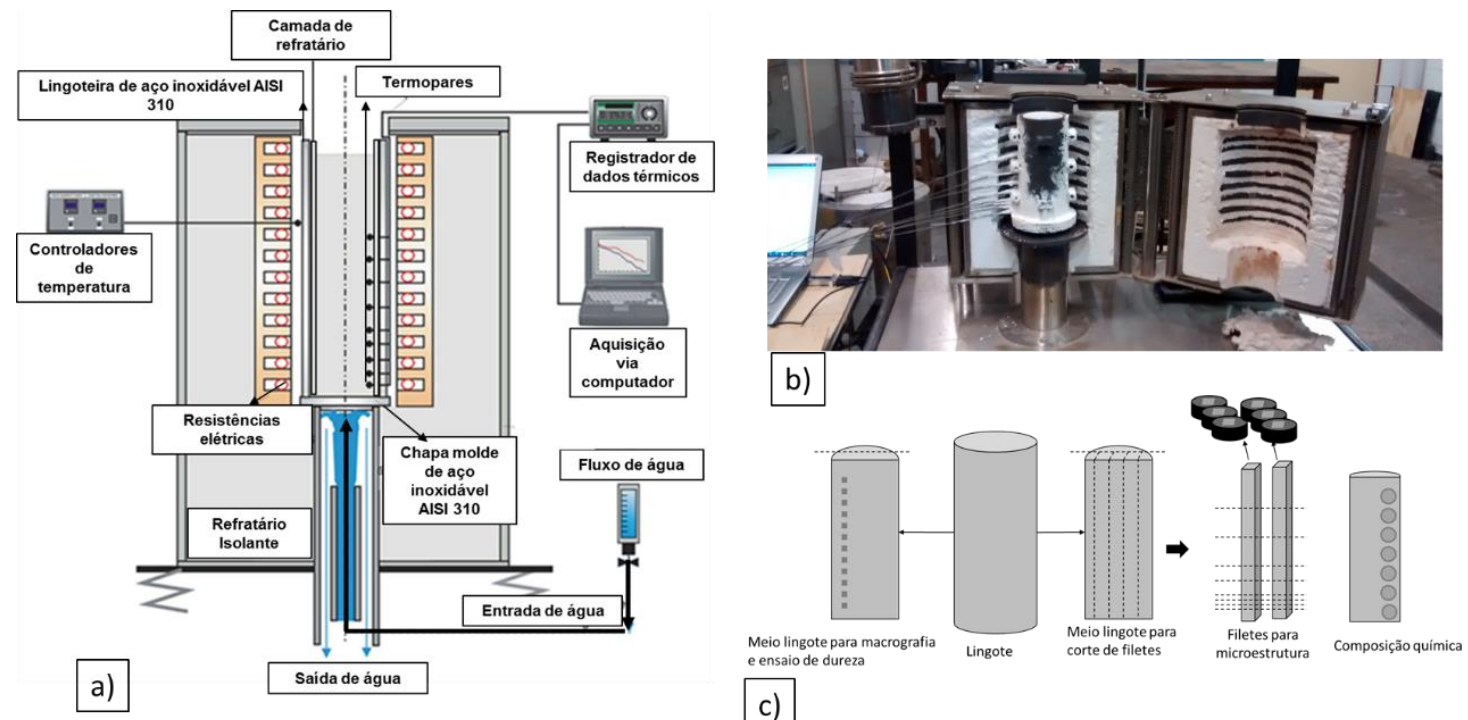

c)

Figura 1 - (a) Dispositivo de solidificação unidirecional vertical ascendente e (b,c) equipamentos envolvidos.

\section{RESULTADOS E DISCUSSÕES}

A composição química do Mg nos três lingotes foi confirmada de acordo com a estequiometria projetada, com $2,4 \%, 2,6 \%$ e 3,2\% em massa. Não houve variações significativas com relação ao teor dos demais elementos de liga uma vez que partiram de um mesmo lote de referência. Não houve variações significativas de teores de elementos ao longo do lingote indicando ausência de qualquer perfil de macrosegregação.

Os resultados das curvas de resfriamentos das três ligas testadas permitiram a obtenção dos valores de $T_{L}$ e $T$ s das três ligas, quais sejam: $T L 5052-2,4 \% M g=643^{\circ} \mathrm{C}$, $\mathrm{T}_{55052-2,4 \% \mathrm{Mg}}=611^{\circ} \mathrm{C}$, TL5052-2,6\%Mg $=640^{\circ} \mathrm{C}$, Ts5052-2,6\%Mg $=607^{\circ} \mathrm{C}, \mathrm{T}_{\mathrm{L}} \mathrm{L} 052-3,2 \% \mathrm{Mg}=638^{\circ} \mathrm{C}$,

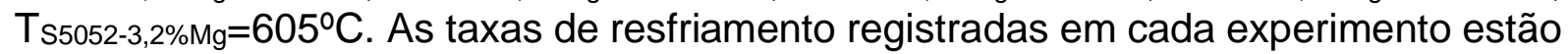
demonstradas nos gráficos da Figura 2. 


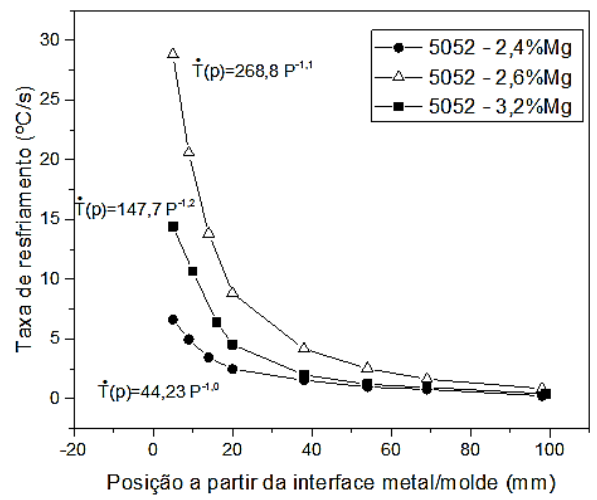

Figura 2 - Evoluções experimentais das taxas de resfriamento obtidas durante a solidificação direcional transiente das ligas 5052 - 2,4\%Mg, 5052 - 2,6\%Mg e 5052 $-3,2 \% \mathrm{Mg}$.

As evoluções das variáveis térmicas foram levantadas a partir da análise das curvas de resfriamento dos termopares acoplados no lingote, conforme a Figura 3 a). Este gráfico permite uma relação entre o tempo de passagem das isotermas $(\mathrm{t})$ liquidus e solidus com a posição ao longo do comprimento do lingote $(P)$, na forma de um gráfico $P(t)=a \cdot t^{b}$. (Figura $3 \mathrm{~b}$ ). A derivada da equação $P(t)$ equivale a velocidade de deslocamento da isoterma liquidus ou da isoterma solidus ao longo do lingote, $V_{L, S}=\frac{d P}{d t}=P^{\prime}(t)=a \cdot b t^{b-1}$. Isolando a variável tempo nas duas equações acima e substituindo uma na outra, podemos obter um gráfico de velocidade em função da posição (posição; $\left.P^{\prime}(t)\right)$ como na Figura 3 c).
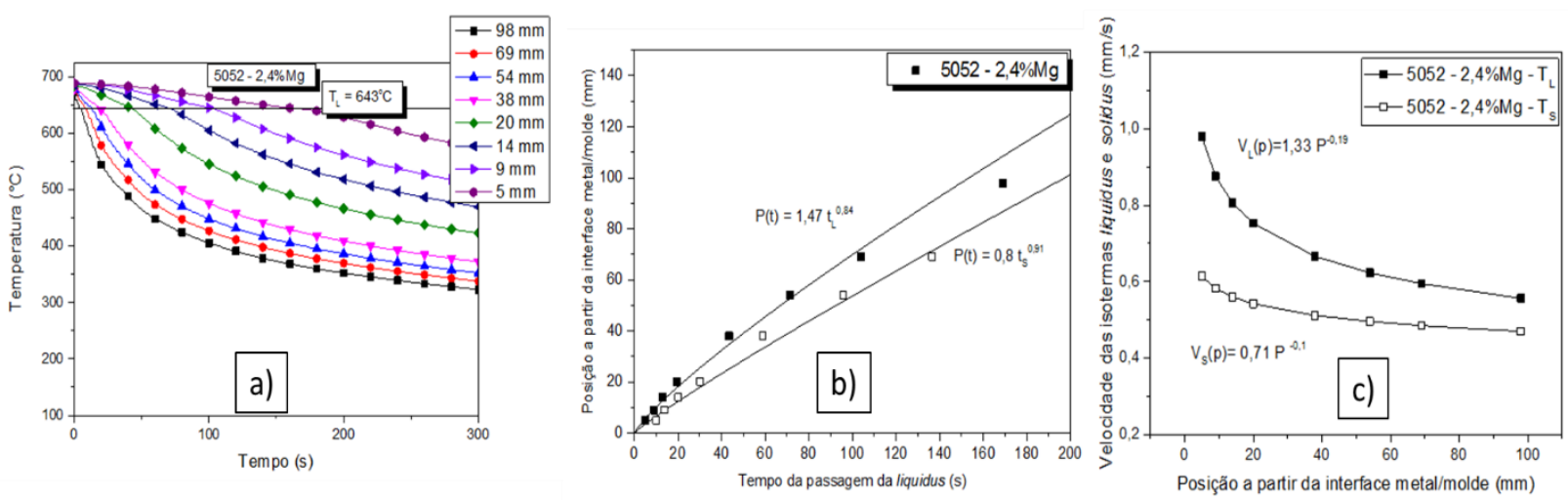

Figura 3 - a) Perfil térmico, b) curvas experimentais dos deslocamentos das posições $(P)$ das isotermas liquidus e solidus em função do tempo de passagem das mesmas, e c) e da velocidade de avanço das isotermas com relação a posição a partir da interface metal/molde, da liga 5052-2,4\%Mg.

O procedimento foi realizado também para as demais ligas, resultando nas seguintes equações para a liga com 2,6\%Mg: $P(t)_{5052-2,6 \% M g}=3,45 t^{\circ}{ }^{0,73}, P(t)_{5052-}$ $2,6 \% M g=2,81 \mathrm{ts}^{0,74}, \quad V_{L}(\mathrm{P})_{5052-2,6 \% M g}=3,9 \mathrm{P}^{-0,36}, \mathrm{~V}_{\mathrm{S}}(\mathrm{P})_{5052-2,6 \% M g}=3,0 \mathrm{P}^{-0,35}$. E para a liga

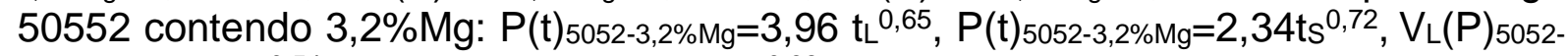

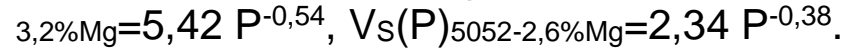

As análises macro e microestruturais das três ligas permitiram observar crescimento predominantemente colunar (Figura 4a) com grãos equiaxiais em posições acima de $110 \mathrm{~mm}$. As Figuras 4b e 4c permitem observar a morfologia dos intermetálicos que tornam-se maiores quanto mais longe da base refrigerada do lingote. As análises de MEV-EDS (Figuras 4h e 4i) acusaram presença de $\mathrm{Mg}$ em 
combinação com Si sendo provavelmente a fase $\mathrm{Mg}_{2} \mathrm{Si}$ formada ao longo da solidificação, ou ainda $\mathrm{Al}_{8} \mathrm{Mg}_{5}$ precipitado a partir da solução solida do alumínio durante o resfriamento após a solidificação [13]. Uma vez que o teor de silício quantificado nas análises de composição química das três ligas foi aproximadamente 0,1\% em massa, é esperado que a maior parte do Mg permaneça em solução sólida. A solubilidade do $\mathrm{Fe}$ em alumínio líquido é muito baixa $(0,052 \%$ em massa, 0,025\% atômica)[14] e portanto todo teor que excede este valor será evidenciado na forma de fases intermetálicas, sendo provavelmente $\mathrm{Al}_{3} \mathrm{Fe}$ com morfologia acicular nos contornos de grão. Podemos inferir ainda presença de fases do sistema Al-Fe-Si, como $\alpha$-AIFeSi, $\beta$-AlFeSi, tipicamente encontrada das ligas comerciais de alumínio, muitas vezes na morfologia de escrita chinesa.

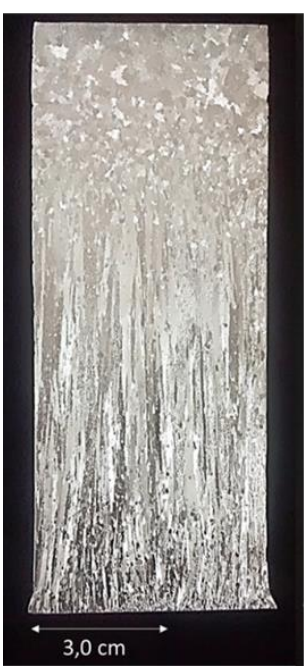

a)
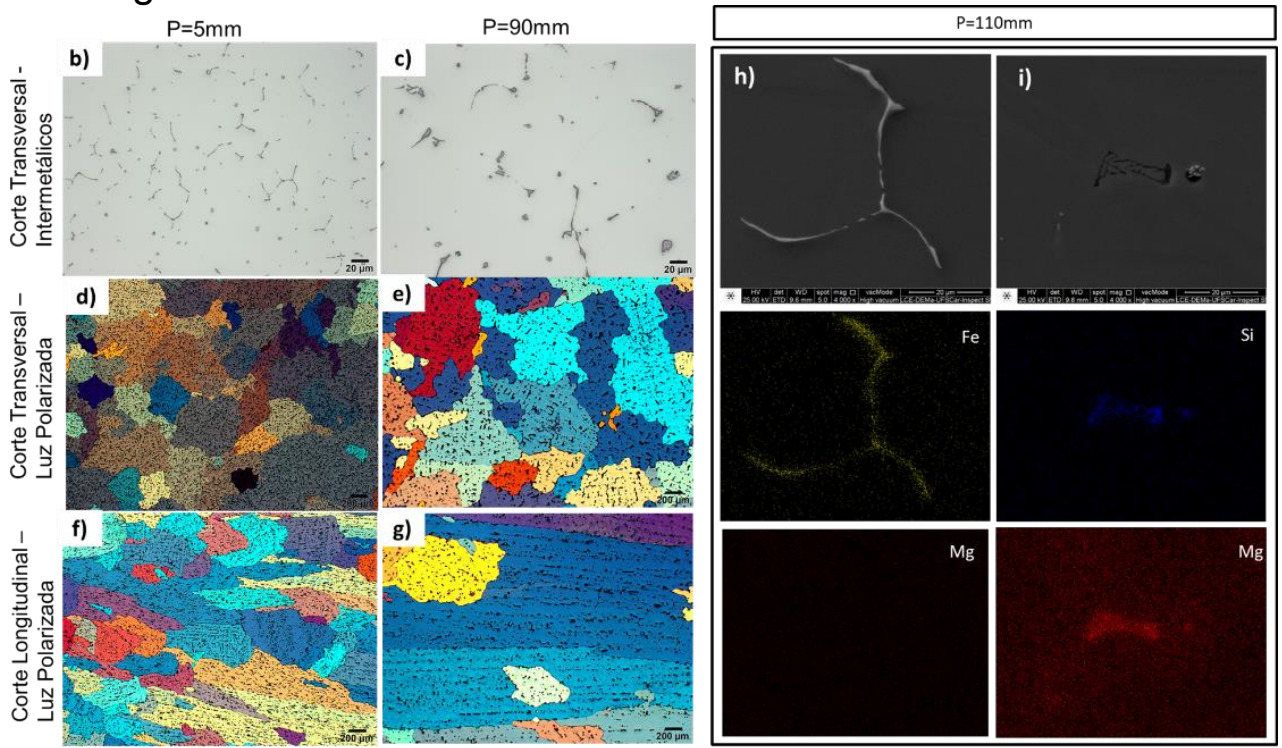

Figura 4 - Caracterização da a) macroestrutura; b), c), d) e), f) e g), da microestrutura e $\mathrm{h}$, i) MEV-EDS das fases presentes na liga $5052-2,4 \% \mathrm{Mg}$.

A análise qualitativa das imagens com luz polarizada (Figuras $4 \mathrm{~d}$, e, f, g) permite observar um comportamento esperado de aumento de tamanho de $\lambda_{1}$ e $\lambda_{2}$ em posições mais distantes da base, correspondendo a taxas de resfriamento inferiores. Não foi possível quantificar os valores de $\lambda_{1}$ da liga $5052-2,6 \% \mathrm{Mg}$ por problemas possivelmente experimentais durante a preparação metalográfica.

A liga $5052-2,4 \% \mathrm{Mg}$ apresentou valores médios de $\lambda_{1}$ variando entre $300 \mu \mathrm{m}$ e $900 \mu \mathrm{m}$, e $\lambda_{2}$ entre $30 \mu \mathrm{m}$ a $70 \mu \mathrm{m}$. A liga $5052-2,6 \% \mathrm{Mg}$ apresentou valores médios de $\lambda_{2}$ menores, variando entre $9 \mu \mathrm{m}$ na posição $P=5 \mathrm{~mm}$, a $25 \mu \mathrm{m}$ em $P=100 \mathrm{~mm}$. Já a terceira liga $5052-3,2 \% \mathrm{Mg}$, analisada até a posição $50 \mathrm{~mm}$ apresentou valores médios de $\lambda_{1}$ entre $160 \mu \mathrm{m}$ e $230 \mu \mathrm{m}$ e de $\lambda_{2}$ entre $6 \mu \mathrm{m}$ e $20 \mu \mathrm{m}$. A magnitude dos espaçamentos dendríticos das três ligas estão em coerência com as taxas de resfriamentos obtidas para cada experimento realizado.

As leis experimentais para evolução microestrutural de $\lambda_{1}$ foram derivadas resultando em $\lambda_{1}=640 \dot{T}_{L}^{-1 / 4}$ para a liga com $2,4 \% \mathrm{Mg}$ e $\lambda_{1}=300 \dot{T}_{L}^{-1 / 4}$ para a liga com 3,2\%Mg. O expoente experimental -1/4 está de acordo com a expressão proposta pro Jackson e Hunt [15] $\left(\lambda_{1}=\right.$ constante $\left.\times \mathrm{V}_{\mathrm{L}}{ }^{-1 / 2}\right)$ modificada pelo modelo analítico de Garcia e Clyne [16]. O expoente -0,55 (Tabela 1) que é normalmente aplicado a ligas binárias não se adequa para representar a evolução de $\lambda_{1} \mathrm{com}$ relação a taxa de resfriamento para esta liga comercial. 
Já as leis de crescimento dendrítico secundário resultaram em expressões $\lambda_{2}=$ $27 V_{L}^{-1,1}$ para a liga $5052-2,4 \% \mathrm{Mg}, \lambda_{2}=15{V_{L}}^{-1,1}$ para as ligas $5052 \mathrm{com} 2,6 \% \mathrm{Mg}$ e $3,2 \% \mathrm{Mg}$.

Nos dois casos $\left(\lambda_{1}\right.$ e $\left.\lambda_{2}\right)$ a adição de $M g$ proporcionou diminuição do espaçamento microestrutural se considerado um mesmo valor de $\mathrm{V}_{\mathrm{L}}$ ou $\dot{T}_{\mathrm{L}}$. No caso das ligas $5052-2,6 \% \mathrm{Mg}$ e $5052-3,2 \% \mathrm{Mg}$ os valores de $\lambda_{2}$ obtidos foram bastante proximos, sendo adotada uma mesma relação de crescimento, conforme mostrado na Erro! Fonte de referência não encontrada.5.
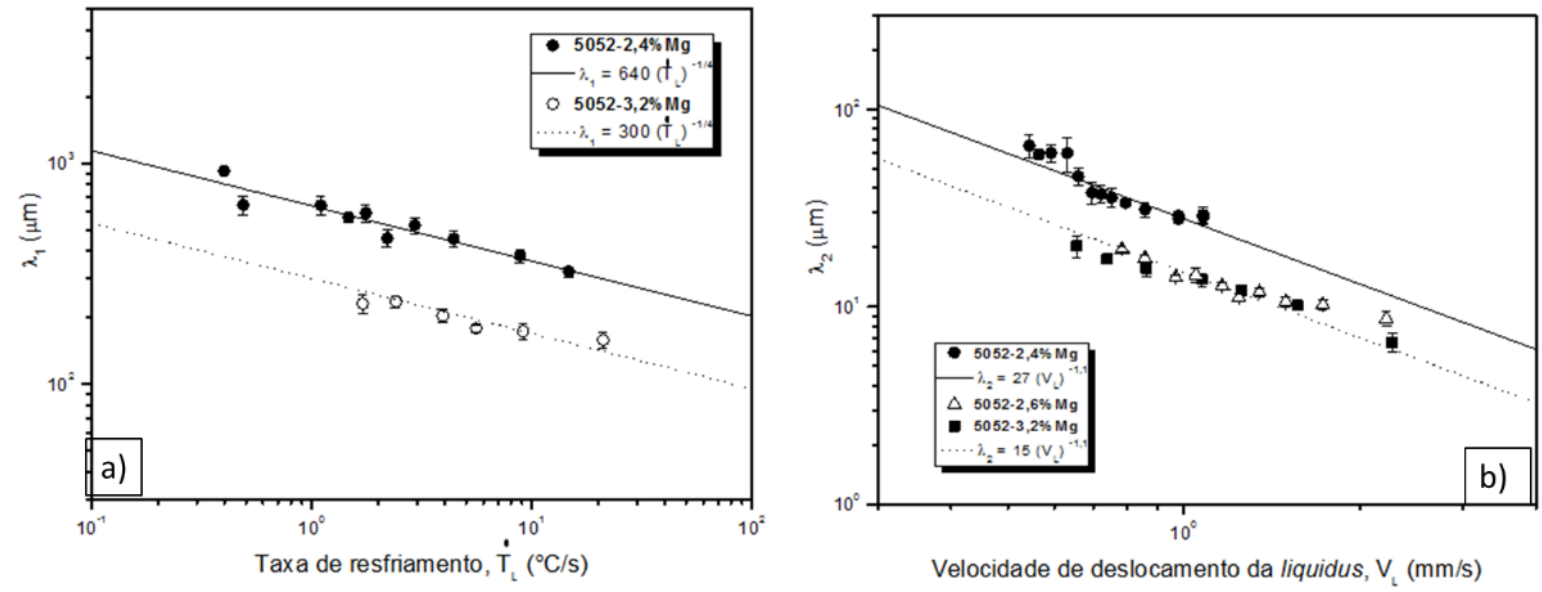

Figura 5 - Leis de crescimento dendrítico a) primário com relação a taxa de resfriamento, b) secundário com relação a velocidade de avanço a isoterma liquidus, das ligas 5052 com teores de 2,4\%, 2,6\% e 3,2\%Mg.

A resistência mecânica das ligas 5052 foi analisada através de ensaios de dureza Brinell, cujos valores foram obtidos em indentações em diferentes posições dos lingotes (Figura 6a). Para as três ligas estudadas, as durezas foram associadas com os valores de espaçamento dendrítico secundário, resultado mostrado na Figura $6 b$.
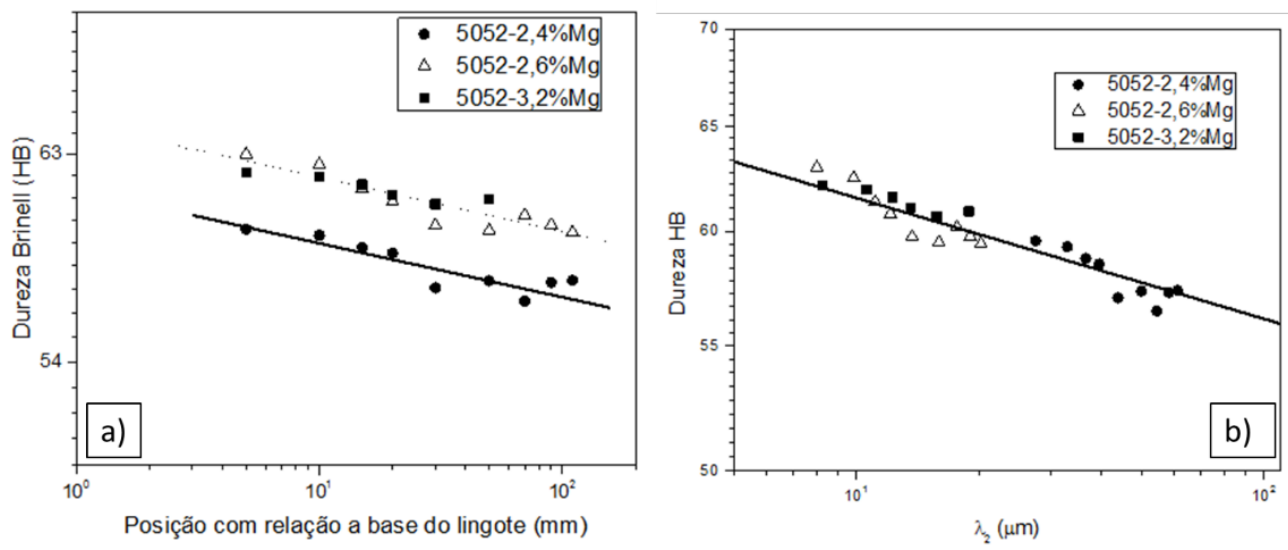

Figura 6 - a) Evolução da dureza Brinell ao longo do comprimento do lingote, e b) evolução da dureza Brinell vs $\lambda_{2}$, das ligas 5052 solidificadas direcionalmente com teores de $2,4 \%, 2,6 \%$ e $3,2 \% \mathrm{Mg}$.

As posições mais próximas da interface metal/molde, em consequência das maiores taxas de resfriamento, apresentaram menores valores de $\lambda_{1}$ e $\lambda_{2}$, e portanto melhor distribuição das fases de reforço mecânico. Nas posições onde as taxas de 
resfriamento foram menores os espaços entre os braços dendríticos são maiores e mais espaçados. Uma mesma área indentada neste último caso possui uma proporção muito menor de fases intermetálicas do que em posições submetidas a altas taxas de resfriamento. Dessa forma, quanto mais próximo da base do lingote, maior é o efeito do tamanho dos intermetálicos na dureza de material. Quanto mais distante da base do lingote, menor é este efeito, tornando a dureza da matriz o fator determinante para medição de tal propriedade. A literatura reporta dureza da ordem de $600-700 \mathrm{HV}$ para as fases de $\mathrm{Mg}_{2} \mathrm{Si}$, e $800-1000 \mathrm{HV}$ para fases do sistema $\mathrm{Fe}-\mathrm{Al}$ $[17,18]$.

A liga contendo $2,4 \%$ de $\mathrm{Mg}$ apresenta menores valores de dureza em relação as diferentes posições monitoradas no lingote e quando comparada com as demais ligas. Isto justifica-se pelos maiores valores de $\lambda_{2}$ caracterizando esta liga. Já quando plotados os valores de dureza $(\mathrm{HB})$ versus $\lambda_{2}$ para as três ligas analisadas uma única relação experimental predomina. $\mathrm{O} \lambda_{2}$ mostra-se como fator predominante no controle da dureza para as ligas 5052 examinadas.

A quantificação da fração em massa dos intermetálicos citados foi realizada através de simulação em Software Thermo-Calc, como mostrado na Figura 7.

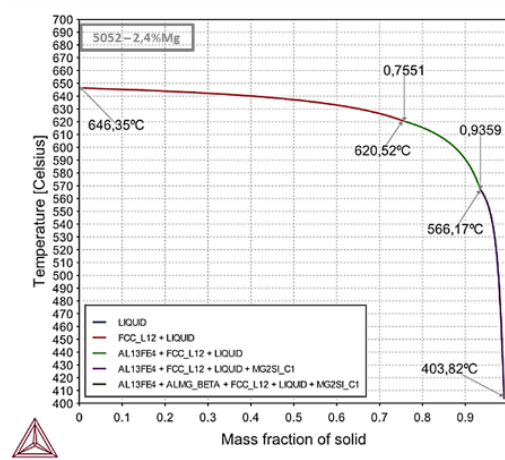

a)

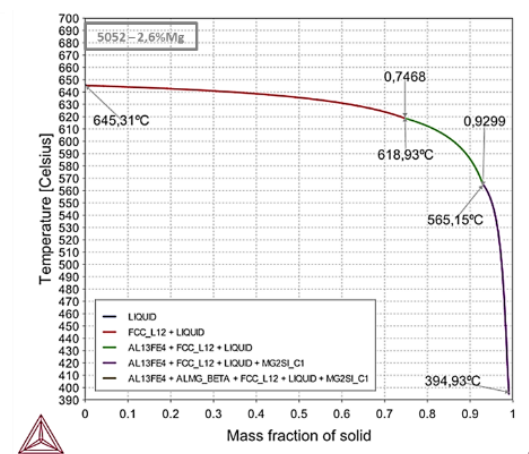

b)

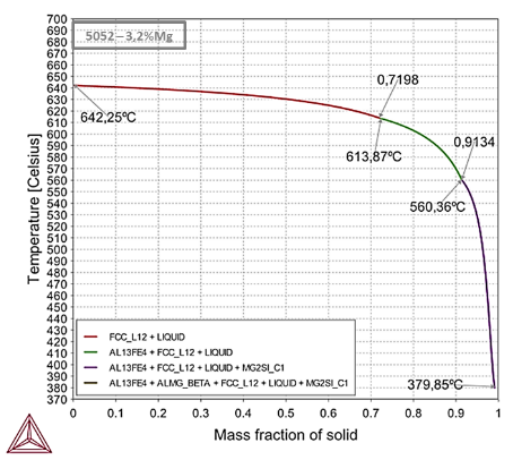

c)

Figura 7 - Resultados das simulações em Thermocalc mostrando gráficos de microssegregação segundo modelo de Scheil das composições da liga 5052 com a) $2,4 \% \mathrm{Mg}$, b) $2,6 \% \mathrm{Mg}$ e c) $3,2 \% \mathrm{Mg}$.

Através dos caminhos de solidificação previstos pela equação de Scheil mostrados nas Figuras 7a), b) e c), é possível observar uma fração ligeiramente maior das fases ricas em Fe e Mg para a liga com 3,2\%Mg (8,7\% contra 7,0\% e 6,4\%). Dessa forma, esta maior fração torna-se mais um ponto de justificativa dos maiores valores de dureza encontrados para a liga com maior teor de $\mathrm{Mg}$. As fases previstas pelo cálculo termodinâmico via software Thermo-Calc basicamente confirmam aquelas observadas por meio de MEV-EDS, anteriormente discutidas.

\section{CONCLUSÕES}

Os experimentos de solidificação unidirecional em regime transiente da liga 5052 foram realizados neste trabalho com objetivo principal de compreender os efeitos da variação de $\mathrm{Mg}$ na evolução de parâmetros térmicos, microestruturais, e variação de dureza Brinell. Com base nos resultados obtidos para as ligas 5052 com 2,4\%, 2,6\% e $3,2 \%$ de $\mathrm{Mg}$, podemos inferir as seguintes conclusões:

- Não foram observadas tendências conclusivas de aumento ou diminuição de da taxa de resfriamento ou velocidade de avanço de solidificação com relação 
a variação de teor de Mg na liga. Há uma dependência muito maior com relação a outras variáveis ao longo do procedimento experimental.

- As leis de crescimento dendrítico primário com relação a taxa de resfriamento obtidas para as ligas com $2,4 \% \mathrm{Mg}$ e $3,2 \% \mathrm{Mg}$ foram respectivamente $\lambda_{1}=$ $640 \dot{T}_{L}^{-1 / 4}$ e $\lambda_{1}=300 \dot{T}_{L}^{-1 / 4}$, cujo expoente experimental -1/4 está de acordo com a expressão proposta por Jackson e Hunt modificada pelo modelo analítico de Garcia e Clyne. O expoente -0,55 que é normalmente aplicado a ligas binárias não se adequa para representar a variação de $\lambda_{1}$ com relação a taxa de resfriamento para esta liga comercial.

- As leis de crescimento dendrítico secundárias com relação a velocidade de solidificação resultaram nas expressões $\lambda_{2}=27 V_{L}^{-1,1}$ para a liga 5052$2,4 \% \mathrm{Mg}$ e $\lambda_{2}=15 V_{L}^{-1,1}$ para as ligas com $2,6 \% \mathrm{Mg}$ e para a liga 5052 $3,2 \% \mathrm{Mg}$.

- A adição de $\mathrm{Mg}$ proporcionou diminuição no espaçamento microestrutural se considerado os mesmos valores de taxa de resfriamento (para $\lambda_{1}$ ) ou velocidade de avanço da isoterma liquidus (para $\lambda_{2}$ ).

- A evolução da dureza está diretamente relacionada ao tamanho e distribuição de intermetálicos, mostrando $\lambda_{2}$ como fator predominante no controle de dureza para as ligas testadas. Quanto menor o valor de $\lambda_{2}$, menores os tamanhos dos intermetálicos e maiores os valores de dureza.

- As principais fases intermetálicas encontradas são ricas em $\mathrm{Fe}, \mathrm{Mg}$ e $\mathrm{Si}$, confirmadas através de análise MEV/EDS, compondo fases de $\mathrm{Mg}_{2} \mathrm{Si}, \mathrm{Al}_{3} \mathrm{Fe}$, $\alpha$-AlFeSi e $\beta$-AlFeSi. A quantificação das fases foi prevista através de simulação com software Thermo-Calc, cujos caminhos de solidificação mostraram uma fração ligeiramente maior das fases ricas em Fe e Mg para a liga com maiores teores de $\mathrm{Mg}(3,2 \%)$, tornando este fato, mais um ponto de justificativa dos maiores valores de dureza encontrados para a liga com maior teor de Mg.

\section{Agradecimentos}

À Companhia Brasileira de Alumínio (CBA) pelo fornecimento de amostras e cooperação com infraestrutura para caracterização microestrutural.

\section{REFERÊNCIAS}

1 BATUR, C. et al. Control of a crystal growth in Bridgman furnace. Journal of Alloys and Compounds, 1995.

2 SILVA, A. P. et al. Microstructural development during transient directional solidification. Materials and Design, v. 31, p. 4584-5491, 2010.

3 L.SILVA, B.; GARCIA, A.; SPINELLI, J. E. The effects of microstructure and intermetallic phases of directionally solidified Al-Fe alloys on microhardness.

Materials Letters, n. 89, p. 291-295, set. 2012.

4 HUNT, J. D.; LU, S. Z. Numerical modeling of cellular array growth: spacing and structure predictions. Mettalurgical and Materials Transactions A, v. 27A, p. 611-623, 1996

5 BOUCHARD, D.; KIRKALDY, J. S. Prediction of dendrite arm spacing in unstedy and steady-state heat flow of unidirectionally binary alloys. Metalurgical and Materials Transaction B, v. 28B, p. 651-633, 1997. 
HUNT, J. D. Keynote Adress: Cellular and primary dendrite spacing.

Proceeding of International Conference on Solidification nad Casting of Metals, London, p. 3-9, 1979.

7 LAPIN, J. E. A. Directional Solidification of Ni-Al-Cr-Fe alloy. Scripta Materialia, v. 37, n. 1, p. 85-91, 1997.

8 GÜDÜZ, M.; ÇADIRLI, E. Directional solidification of Aluminium-cooper alloys. Materials Science and Engineering A, v. 327, n. 2, p. 167-185, 2002.

9 QUARESMA, J. M. V.; SANTOS, C. A.; GARCIA, A. Correlation between Unsteady-State Solidification Condition, Dendrite Spacing and Mechanical Propoerties of Al-Cu Alloys. Metallurgical and Materials Transactions A, v. 31A, p. 3167-3178, 2000.

10 GARCIA, A. Solidificações: Fundamentos e aplicações. 2. ed. Campinas: Editora da Unicamp, 2007.

11 BRITO, C. C. D. Parâmetros Térmicos e Microestruturais na Solidificação Transitória de Ligas Al-Mg e Al-Mg-Si e Correlação com Resistências Mecânica e à Corrosão. Tese de Doutorado (UNICAMP). Campinas. 2016.

12 GÜNDÜZ, M.; E.ÇADıRLı. Directional solidification of aluminium-copper alloys. Materials Science and Engineering A327, p. 167-185, 2002.

13 N.A.BELOV; ESKIN, D. G.; A.A.AKSENOV. Multicomponent Phase Diagrams: Apllication for Commercial Aluminum Alloys. Amsterdam: Elsevier, 2005.

14 J.E.HATCH; MACKENSIE, D. S. Handbook of Aluminum: Physical Metallurgy and Properties. New York: Marcel Dekker, Inc. , v. 1, 2003.

15 JACKSON, K. A.; HUNT, J. D. Lamellar and Rod Eutetic Growth. Transactions os the Metalurgical Society of AIME, v. 236, p. 1129-1142, 1966.

16 CLYNE, T. W.; GARCIA, A. The application of a new solidification heat flow model to splat cooling. Jounal of Materials Science, v. 16, p. 1643-1653, 1981.

$17 \mathrm{KONDOH}$, et al. In-situ Synthesis of Mg2Si Intermetallics via Powder Metallurgy Process. Materials Transactions, v. 44, n. 5, p. 981-985, 2003.

18 MONDOLFO, L. F. Aluminium Alloys: Structure and Properties. Butterworths, London, 1979. 